\title{
A rapid method to screen wild Solanum for resistance to early blight
}

\author{
Pieter J. Wolters • Lennard de Vos • Gerard Bijsterbosch • Joyce H. C. Woudenberg • \\ Richard G. F. Visser • Gerard van der Linden • Vivianne G. A. A. Vleeshouwers
}

Accepted: 4 April 2019/Published online: 17 April 2019

(C) The Author(s) 2019

\begin{abstract}
Early blight of potato and tomato is caused by Alternaria fungi and negatively impacts crop yields. Environmental factors and plant maturity influence disease development, which is usually kept under control by fungicide applications. Wild tuber-bearing Solanum section Petota species are a promising source of resistance to early blight that could be used to control the disease, for example by crossbreeding or modern breeding approaches. An efficient screening method is a first prerequisite for the identification of resistant genotypes in wild Solanum germplasm. Here, we describe a protocol that can be used to rapidly screen for resistance to early blight in wild Solanum collections. This protocol provides a good starting point for the identification of resistant genotypes and is a step towards breeding for resistance to early blight using wild Solanum species.
\end{abstract}

Keywords Alternaria $\cdot$ A. grandis $\cdot$ A. protenta . A. solani $\cdot$ Disease assay $\cdot$ Wild potato

P. J. Wolters · L. de Vos · G. Bijsterbosch • R. G. F. Visser • G. van der Linden • V. G. A. A. Vleeshouwers $(\bowtie)$ Wageningen UR Plant Breeding, Wageningen University and Research, Droevendaalsesteeg 1, 6708 PBWageningen, The Netherlands

e-mail: vivianne.vleeshouwers@wur.nl

J. H. C. Woudenberg

Westerdijk Fungal Biodiversity Institute, Uppsalalaan 8, 3584

CTUtrecht, The Netherlands
Alternaria fungi are associated with diseases in potato. Small-spored Alternaria species such as A. alternata and $A$. arborescens can cause brown leaf spot disease (van der Waals et al. 2011; Tymon et al. 2016a; Tymon et al. 2016b), while large-spored A. solani, A. grandis and $A$. protenta can cause early blight (Duarte et al. 2014; Woudenberg et al. 2014; Bessadat et al. 2015; Ayad et al. 2017). Early blight is characterised by the appearance of dark necrotic lesions on infected leaves, which are often restricted within the leaf veins and have a typical concentric ring pattern. Affected leaves have a reduced photosynthetic potential and severe infections lead to defoliation of the plant, which negatively influences crop yield (Johnson and Teng 1990; Rotem 1994; Shtienberg et al. 1996).

Paul Sorauer first described early blight in Europe and considered $A$. solani a weak pathogen with a mainly saprophytic lifestyle that only infects plants with the right predisposition under especially favourable conditions (Sorauer 1896). He recognised the importance of environmental and host factors in disease development at a time when the attention of most plant pathologists was focused on the pathogens and when almost all fungi that occur on plants were considered parasites (Westerdijk 1917).

Many studies have since confirmed that early blight development is greatly influenced by factors such as temperature, humidity, leaf wetness and abiotic stress, or by the occurrence of other plant diseases (Holley et al. 1985; Bussey and Stevenson 1991; Rotem 1994). Genetic host factors also contribute to resistance to early blight in potato (Herriott et al. 1990; Christ and Haynes 
2001; Xue et al. 2019). It was found that early maturing potato cultivars are generally more susceptible to early blight than late maturing cultivars, but no fully resistant cultivars have been identified (Rodriguez et al. 2006; Duarte et al. 2014; Odilbekov 2015; Abuley et al. 2018; Xue et al. 2019).

Early blight control is currently based on the application of fungicides (Abuley and Nielsen 2017), but various studies have reported a reduced efficacy of the chemicals that are being used (Pasche et al. 2005; Rosenzweig et al. 2008; Fairchild et al. 2013). Moreover, the use of fungicides presents a cost for farmers and could lead to adverse environmental effects.

Some wild potato relatives show high levels of resistance and could possibly provide an alternative solution to control early blight (Jansky et al. 2008; Weber and Jansky 2012; Meier et al. 2015; Haynes and Qu 2016). Such genotypes could be used in classical breeding, but could also provide a starting point to identify genes that contribute to resistance for use in modern breeding approaches.

An efficient screening method is required for testing large numbers of genotypes from wild germplasm collections for resistance to early blight. Several protocols for testing early blight resistance have already been described by others (Bussey and Stevenson 1991; Jansky et al. 2008; Weber and Jansky 2012; Odilbekov et al. 2014), but not all methods are suitable for screening wild Solanum species. Such species are very diverse and may respond differently to the varying environmental conditions that are encountered in a field. We have therefore developed a greenhouse protocol, based on previously described methods (Rodrigues et al. 2010; Odilbekov et al. 2014), in which environmental conditions are controlled to favour early blight infection. This allows us to properly evaluate the role of genetic factors in resistance. The protocol is optimised for the fast characterisation of plants that are maintained in vitro.

Potato (S. tuberosum cv. Désirée) plants and wild Solanum genotypes (listed in Table 1) with various levels of resistance to early blight were propagated in vitro on MS20 medium (4.4 g/l Murashige and Skoog basal salt mixture including vitamins, $20 \mathrm{~g} / 1$ sucrose, and $8 \mathrm{~g} / \mathrm{l}$ micro agar in water) (Murashige and Skoog 1962). Two weeks after propagation, plants were transferred to $11 \times 11 \times 12 \mathrm{~cm}$ pots with sterilized soil and grown in a climate regulated greenhouse under long-day conditions $\left(16 \mathrm{~h}\right.$ light and $22{ }^{\circ} \mathrm{C}, 8 \mathrm{~h}$ dark and $18{ }^{\circ} \mathrm{C}$ ) with sufficient space in between to allow adequate light exposure.

Five isolates belonging to three different species of Alternaria from different locations were used in this study (Table 2). We have previously described A. solani isolate CBS 143772 (Iftikhar et al. 2017; Wolters et al. 2018) and additional Alternaria isolates were ordered from the fungal culture collection (CBS) of the Westerdijk Institute (Utrecht, the Netherlands). The Alternaria isolates were maintained on potato dextrose agar (Beever and Bollard 1970) in Petri dishes.

To produce conidia, agar plugs containing mycelium of the Alternaria isolates were used to inoculate V8 medium ( $5 \mathrm{x}$ diluted V8 juice), followed by incubation on a shaking incubator at $28{ }^{\circ} \mathrm{C}$ until the colour of the culture changed from red to almost black after 4-5 days. The Alternaria culture was then poured directly into Petri dishes containing potato dextrose agar and incubated without lid in an incubator equipped with blacklight fluorescent tubes (Philips TL-D $18 \mathrm{~W}$ BLB) at $25{ }^{\circ} \mathrm{C}(12 \mathrm{~h}$ dark, $12 \mathrm{~h}$ blacklight $)$ for 3 days. The plates were covered and stored at $25^{\circ} \mathrm{C}$ in the dark until needed.

Five-week old plants were transferred to a climate cell $\left(25^{\circ} \mathrm{C}\right.$, relative humidity $>70 \%, 16 \mathrm{~h}$ light and $8 \mathrm{~h}$ dark) one day before inoculation. The plants were placed inside a tent that was constructed by covering a frame with transparent plastic sheeting. An atomiser (Condair 505) was placed inside the tent together with the plants, to create a fine mist and increase humidity after inoculation of the plants (Fig. 1a).

On the day of the inoculation, conidia were harvested by flooding the Alternaria cultures with tap water and gently brushing the surface. Next, the conidial suspension was filtered through a tea strainer to remove pieces of mycelium and the conidia were left to sink so the bottom. Supernatant was poured off and the conidia were resuspended in $1 / 5$ strength potato dextrose broth

Table 1 Solanum genotypes used in this study

\begin{tabular}{ll}
\hline Genotype & Solanum species \\
\hline PLD 782-9 & S. polyadenium \\
IMT 63-3 & S. immite \\
PLD 207-1 & S. polyadenium \\
Désirée & S. tuberosum \\
GIG 715-1 & S. microdontum subsp. gigantophyllum \\
\hline
\end{tabular}


Table 2 Alternaria isolates used in this study

\begin{tabular}{llll}
\hline Accession & Alternaria species & Original host & Origin \\
\hline CBS 143772 & A. solani & Solanum tuberosum & Netherlands \\
CBS 109157 & A. solani & Solanum tuberosum & USA \\
CBS 116695 & A. grandis & Solanum tuberosum & USA \\
CBS 116437 & A. protenta & Hordeum vulgare & New Zealand \\
CBS 116696 & A. protenta & Helianthus annuus & Israel \\
\hline
\end{tabular}

(PDB) supplemented with $0.3 \%$ micro-agar to obtain a suspension of $1 \times 10^{5}$ conidia $/ \mathrm{ml}$. The agar was added to increase the density of the spore suspension, leading to firmer droplets that do not easily run off the inoculated leaves. Diluted PDB was used to make sure that the conidia germinate consistently (Fig. 1b).

The upper three fully expanded compound leaves (further sub-classified as 'upper', 'middle' and 'lower' leaves) of the plants were drop-inoculated with $10 \mu \mathrm{l}$ droplets of conidial suspension. It should be noted that wild Solanum genotypes are morphologically very diverse. Therefore, it can be difficult to pick comparable leaves for inoculation. Depending on the size of the leaves of the genotype tested, 4-6 droplets can be used to inoculate each compound leaf. For each genotype, three replicates were included in the experiment and the experiment was repeated once. Both experiments were carried out at the Laboratory of Plant Breeding at Wageningen University (Wageningen, the Netherlands) in 2016.

Directly after inoculating the plants, lights were switched off and the atomiser was turned on. The normal light regime was resumed the next day and the atomiser was only switched on during subsequent nights. This mimics the alternating leaf wetness during the night and gradual drying of the leaves during the course of the day that can occur outdoors. Figure 1c shows an example of the lesions that are produced on the second fully expanded leaf from the top of potato cultivar Désirée, 5 days after inoculation with six droplets of spore suspension. In the experiment that is summarized in Figs. 2 and 3, we have used four droplets of spore suspension to inoculate each leaf (12 droplets per plant). Diameters of lesions produced on all the inoculated spots were recorded 5 days after inoculation using a digital calliper (Mitutoyo 500-161-30). The averages of the lesion sizes formed by each isolate on the different Solanum genotypes are summarized in Fig. 2.
The disease protocol that is described here differs in several aspects from the protocol described by Odilbekov et al. (2014). We start with in vitro maintained plants, which allows the testing of wild Solanum germplasm. By using an atomiser, leaf wetness at night is ensured. Combined with using an increased spore concentration of $1 \times 10^{5}$ spores $/ \mathrm{ml}$, consistent lesion development is achieved, allowing the measuring of lesions at 5 days post inoculation already.

The tested Solanum genotypes ranged from resistant to susceptible, with average lesion sizes of less than $1 \mathrm{~mm}$ in the most resistant genotype ( $S$. polyadenium 207-1), to over $10 \mathrm{~mm}$ in susceptible genotypes (S. tuberosum cv. Désirée and S. microdontum subsp. gigantophyllum 715-1, Fig. 2). While the effects of maturity are likely to be less pronounced in young plants, they cannot be fully eliminated. When recording the lesion sizes, it is important to distinguish between the different positions of the leaves that are inoculated. A pronounced effect of leaf position on lesion size was seen in the susceptible genotypes, pointing to an effect of leaf maturity on early blight development (Fig. 3), as was also observed previously by others (Rodriguez et al. 2006; Odilbekov et al. 2014). Interestingly, this effect seems to be absent in the more resistant genotypes (S. polyadenium 207-1 and 782-9 and S. immite 63-3, Fig. 2).

The method for spore production that was used by us is based on the protocol described by Rodrigues et al. (2010). We omitted the grinding step, but did expose the mycelium to UV light and drought stress. Spores were produced within 10 days for all isolates. The tested isolates were all found to be pathogenic on potato cultivar Désirée, but were shown to differ in virulence. CBS 116695 (A. grandis) appeared to be the most virulent of the isolates that were tested, whereas CBS 116696 (A. protenta) showed the lowest virulence in the assay (Fig. 2). CBS 143772 (A. solani) displayed an 


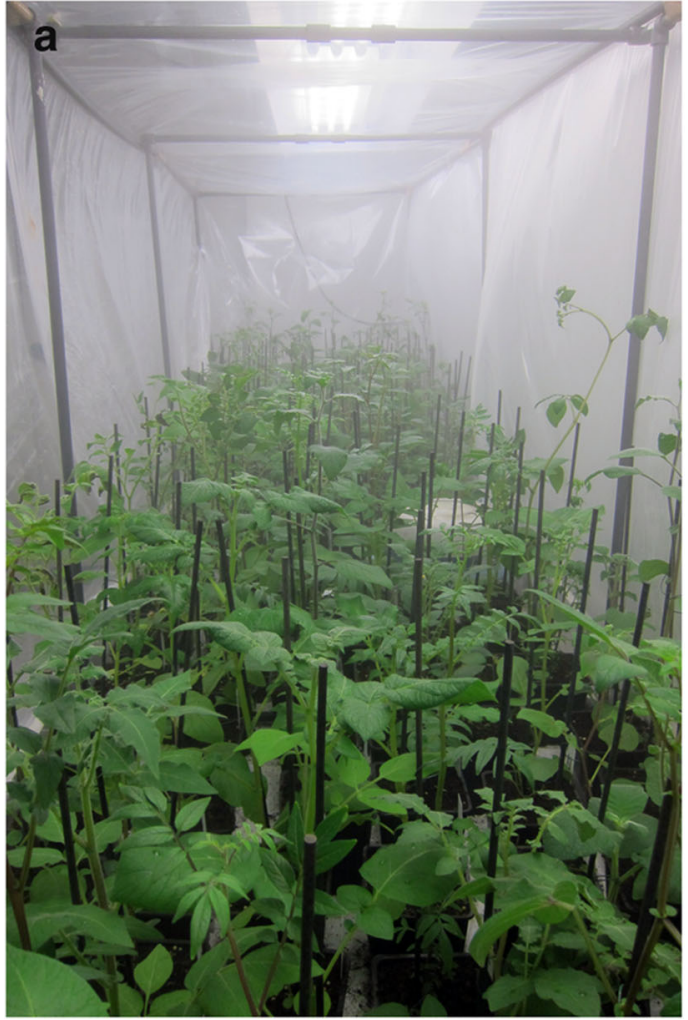

Fig. 1 Setup of the disease test (a), pictures of germinating spores (b) and lesions produced by Alternaria solani (c). a. Plants were incubated inside a transparent plastic tent and an atomiser was placed inside the tent to create a fine mist. b. Addition of potato dextrose to the inoculation medium results in high spore germination efficiency, as evidenced by abundant
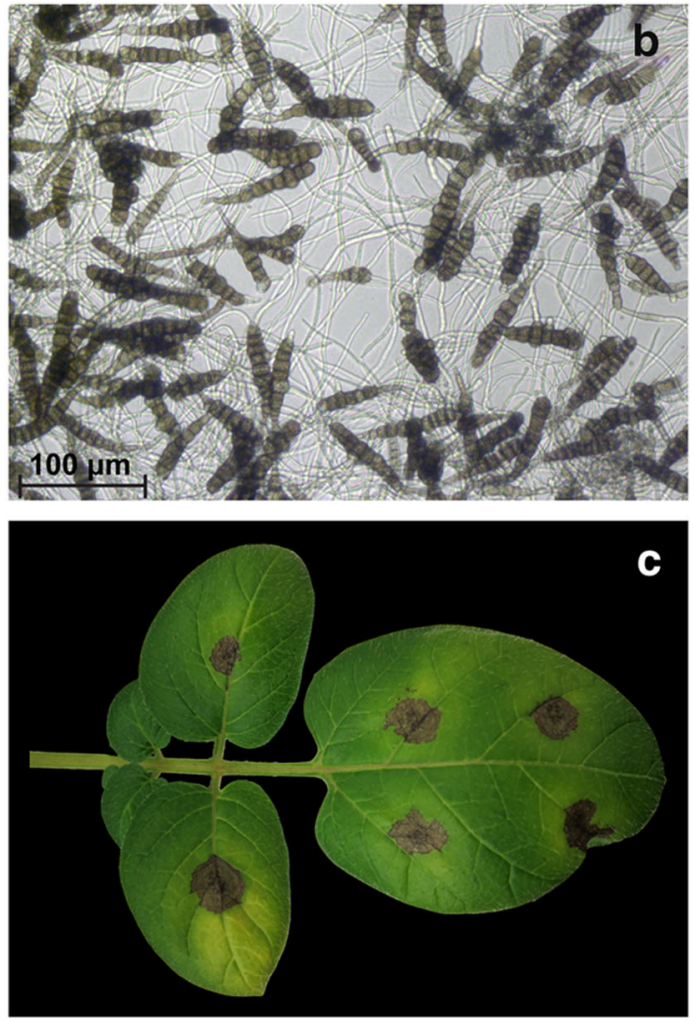

mycelial growth $6 \mathrm{~h}$ after resuspending the spores. $\mathbf{c}$. The adopted inoculation protocol results in consistent lesion development. A representative photograph of a middle leaf of Solanum tuberosum cultivar Désirée inoculated with six droplets of Alternaria solani spore suspension was taken five days post inoculation

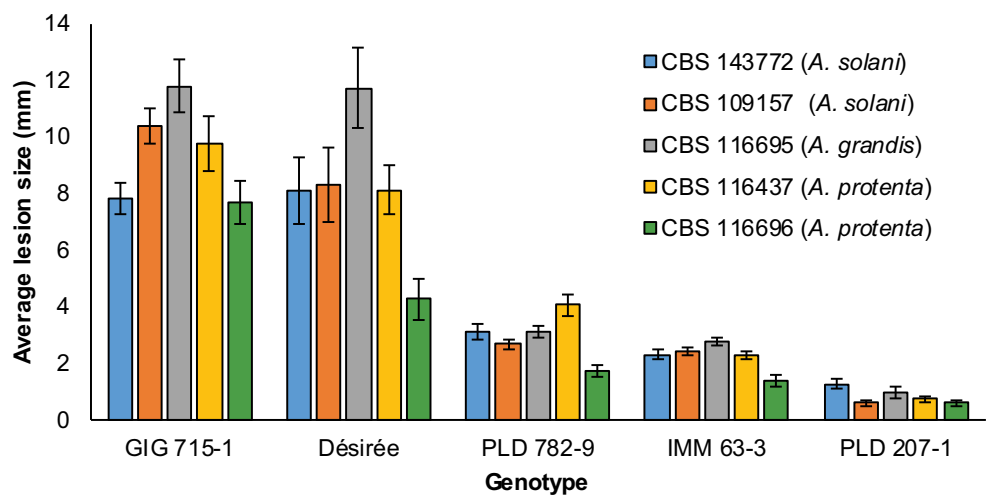

Fig. 2 Pathogenicity of different Alternaria isolates on Solanum genotypes. Average lesion sizes (at five days post inoculation) produced by five different Alternaria isolates/species on different Solanum genotypes (S. microdontum subsp. gigantophyllum 715-1, S. tuberosum cv. Désirée, S. polyadenium 782-9 and S. immite 63-3 and S. polyadenium 207-1, all leaf positions combined) are displayed. Error bars indicate standard error of the mean. All isolates are pathogenic on cultivated potato (Solanum tuberosum cultivar Désirée), but display differences in virulence. No difference in resistance pattern (ranking of Solanum genotypes from susceptible to resistant) is observed among the isolates tested 


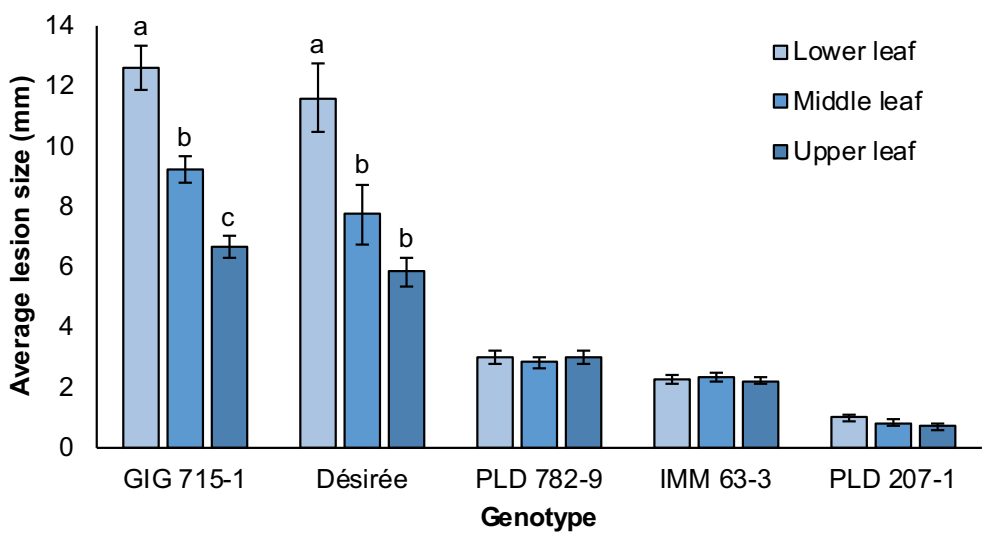

Fig. 3 Effect of leaf position on lesion size. The data for the different isolates and the two experiments are combined and average lesion sizes (at five days post inoculation) produced on lower, middle and upper leaves of different Solanum genotypes are displayed. Error bars indicate standard error of the mean. ANOVA showed a significant effect $(p<0.05)$ of leaf position on lesion size

intermediate virulence. Despite the differences in virulence, the resistance pattern of the different Solanum genotypes was the same for all isolates (Fig. 2), which illustrates the reliability of the protocol.

It can be expected to find large variations in susceptibility or resistance to early blight in Solanum germplasm. Once promising resistant genotypes have been identified, a more detailed characterization of the resistance would be desired. For example, it would be useful to investigate the disease progress over time to get insight into the effect of maturity, investigate the effect of abiotic and other biotic stresses, test performance in the field, and test additional Alternaria isolates. These experiments are especially important when the goal is to introduce resistance in a potato cultivar, to make sure that the targeted resistance is of practical use.

The protocol that is described here will be useful for carrying out resistance screens and can provide a good starting point for subsequent studies. Such follow-up studies will help to clarify the mechanisms that underlie resistance or susceptibility to early blight and, ultimately, to develop resistant potato cultivars.

Acknowledgements We would like to thank the J.R. Simplot Company for funding this research and Nicolas Champouret for providing feedback on the manuscript.

Author's contributions Planned the experiments: PJW, LdV, GB, RGFV, GvdL, VGAAV. Performed the experiments: PJW, LdV, GB. Analysed the data: PJW, LdV. Contributed reagents/ for S. microdontum subsp. gigantophyllum 715-1 and S. tuberosum cv. Désirée and the effect of leaf position on lesion size is analysed separately for these genotypes. Different letters are used to indicate significant differences between the average lesions size produced on leaves of different positions (Tukey's HSD test, $\alpha=0.05)$

materials/analysis tools: JHCW. Wrote the manuscript: PJW, RGFV, VGAAV.

\section{Compliance with ethical standards}

Conflict of interest The authors state they have no conflict of interest.

Human and animal rights Research did not involve Human Participants and/or Animals.

Informed consent Informed consent was obtained from all individual participants included in the study.

Open Access This article is distributed under the terms of the Creative Commons Attribution 4.0 International License (http:// creativecommons.org/licenses/by/4.0/), which permits unrestricted use, distribution, and reproduction in any medium, provided you give appropriate credit to the original author(s) and the source, provide a link to the Creative Commons license, and indicate if changes were made.

\section{References}

Abuley, I. K., \& Nielsen, B. J. (2017). Evaluation of models to control potato early blight (Alternaria solani) in Denmark. Crop Protection, 102, 118-128. https://doi.org/10.1016/j. cropro.2017.08.012.

Abuley, I. K., Nielsen, B. J., \& Labouriau, R. (2018). Resistance status of cultivated potatoes to early blight (Alternaria solani) in Denmark. Plant Pathology, 67(2), 315-326. 
Ayad, D., Leclerc, S., Hamon, B., Kedad, A., Bouznad, Z., \& Simoneau, P. (2017). First report of early blight caused by Alternaria protenta on potato in Algeria. Plant Disease, 101(5), 836. https://doi.org/10.1094/pdis-10-16-1420-pdn.

Beever, R. E., \& Bollard, E. G. (1970). The nature of the stimulation of fungal growth by potato extract. Microbiology, 60(2), 273-279. https://doi.org/10.1099/00221287-60-2273.

Bessadat, N., Hamon, B., Henni, D. E., \& Simoneau, P. (2015). First report of tomato early blight caused by Alternaria grandis in Algeria. Plant Disease, 100(2), 533-533. https://doi.org/10.1094/pdis-05-15-0613-pdn.

Bussey, M., \& Stevenson, W. (1991). A leaf disk assay for detecting resistance to early blight caused by Alternaria solani in juvenile potato plants. Plant Disease, 75(4), 836-390.

Christ, B. J., \& Haynes, K. (2001). Inheritance of resistance to early blight disease in a diploid potato population. Plant Breeding, 120(2), 169-172.

Duarte, H. S., Zambolim, L., Rodrigues, F. A., Paul, P. A., Pádua, J. G., Ribeiro Júnior, J. I., et al. (2014). Field resistance of potato cultivars to foliar early blight and its relationship with foliage maturity and tuber skin types. Tropical Plant Pathology, 39(4), 294-306.

Fairchild, K. L., Miles, T. D., \& Wharton, P. S. (2013). Assessing fungicide resistance in populations of Alternaria in Idaho potato fields. Crop Protection, 49, 31-39.

Haynes, K. G., \& Qu, X. (2016). Late blight and early blight resistance from Solanum hougasii introgressed into Solanum tuberosum. American Journal of Potato Research, 93(1), 86-95.

Herriott, A., Haynes, F., \& Shoemaker, P. (1990). Inheritance of resistance to early blight disease in tetraploid $\times$ diploid crosses of potatoes. HortScience, 25(2), 224-226.

Holley, J., Hall, R., \& Hofstra, G. (1985). Effects of cultivar resistance, leaf wetness duration and temperature on rate of development of potato early blight. Canadian Journal of Plant Science, 65(1), 179-184.

Iftikhar, S., Shahid, A. A., Halim, S. A., Wolters, P. J., Vleeshouwers, V., \& Ahmad, S. (2017). Discovering novel Alternaria solani succinate dehydrogenase inhibitors by in silico modeling and virtual screening strategies to combat early blight. Frontiers in Chemistry, 5, 100.

Jansky, S., Simon, R., \& Spooner, D. (2008). A test of taxonomic predictivity: Resistance to early blight in wild relatives of cultivated potato. Phytopathology, 98(6), 680-687.

Johnson, K., \& Teng, P. (1990). Coupling a disease progress model for early blight to a model of potato growth. Phytopathology, $80(4), 416-425$.

Meier, A., Jansky, S., \& Halterman, D. (2015). Germplasm release: Three potato clones incorporating combined resistances to early blight from S. palustre and late blight from $\mathrm{S}$. bulbocastanum into a S. tuberosum background. American Journal of Potato Research, 92(3), 410-416.

Murashige, T., \& Skoog, F. (1962). A revised medium for rapid growth and bio assays with tobacco tissue cultures. Physiologia Plantarum, 15(3), 473-497.

Odilbekov, F. (2015). Resistance to early blight in potato and genetic structure of the pathogen population in Southeast Sweden. $\mathrm{PhD}$ thesis, Alnarp (SE).
Odilbekov, F., Carlson-Nilsson, U., \& Liljeroth, E. (2014). Phenotyping early blight resistance in potato cultivars and breeding clones. Euphytica, 197(1), 87-97. https://doi. org/10.1007/s10681-013-1054-4.

Pasche, J. S., Piche, L. M., \& Gudmestad, N. C. (2005). Effect of the F129L mutation in Alternaria solani on fungicides affecting mitochondrial respiration. Plant Disease, 89(3), 269278. https://doi.org/10.1094/pd-89-0269.

Rodrigues, T. T. M. S., Maffia, L. A., Dhingra, O. D., \& Mizubuti, E. S. G. (2010). In vitro production of conidia of Alternaria solani. Tropical Plant Pathology, 35, 203-212.

Rodriguez, D., Brommonschenkel, S., Matsuoka, K., \& Mizubuti, E. (2006). Components of resistance to early blight in four potato cultivars: Effect of leaf position. Journal of Phytopathology, 154(4), 230-235.

Rosenzweig, N., Atallah, Z., Olaya, G., \& Stevenson, W. (2008). Evaluation of QoI fungicide application strategies for managing fungicide resistance and potato early blight epidemics in Wisconsin. Plant Disease, 92(4), 561-568.

Rotem, J. (1994). The genus Alternaria: Biology, epidemiology, and pathogenicity. American Phytopathological Society.

Shtienberg, D., Blachinsky, D., Ben-Hador, G., \& Dinoor, A. (1996). Effects of growing season and fungicide type on the development of Alternaria solani and on potato yield. Plant disease (USA).

Sorauer, P. (1896). Auftreten einer dem amerikanischen „Early blight" entsprechenden Krankheit an den deutschen Kartoffeln. Zeitschrift für Pflanzenkrankheiten, 6(1), 1-9.

Tymon, L. S., Cummings, T. F., \& Johnson, D. A. (2016a). Pathogenicity and aggressiveness of three Alternaria spp. on potato foliage in the US northwest. Plant Disease, 100(4), 797-801.

Tymon, L. S., Peever, T. L., \& Johnson, D. A. (2016b). Identification and enumeration of small-spored Alternaria species associated with potato in the US northwest. Plant Disease, 100(2), 465-472.

van der Waals, J. E., Pitsi, B. E., Marais, C., \& Wairuri, C. K. (2011). First report of Alternaria alternata causing leaf blight of potatoes in South Africa. Plant Disease, 95(3), 363-363. https://doi.org/10.1094/PDIS-11-10-0820.

Weber, B., \& Jansky, S. (2012). Resistance to Alternaria solani in hybrids between a Solanum tuberosum haploid and $\mathrm{S}$. raphanifolium. Phytopathology, 102(2), 214-221.

Westerdijk, J. (1917). Nieuwe wegen van het phytopathologisch onderzoek. Inaugural lecture, Utrecht (NL).

Wolters, P. J., Faino, L., van den Bosch, T., Evenhuis, B., Visser, R., Seidl, M. F., et al. (2018). Gapless genome assembly of the potato and tomato early blight pathogen Alternaria solani. Molecular Plant-Microbe Interactions, 31, 692-694. https://doi.org/10.1094/mpmi-12-17-0309-a.

Woudenberg, J. H., Truter, M., Groenewald, J. Z., \& Crous, P. W. (2014). Large-spored Alternaria pathogens in section Porri disentangled. Studies in Mycology, 79, 1-47. https://doi. org/10.1016/j.simyco.2014.07.003.

Xue, W., Haynes, K. G., \& Qu, X. (2019). Characterization of early blight resistance in potato cultivars. Plant Disease, PDIS-05-18-0794-RE, PDIS-05-18-0794. 